\title{
IMPACT OF NUCLEAR DATA LIBRARIES ON PREDICTED FAST REACTOR PERFORMANCE
}

\author{
A.G. Nelson ${ }^{1}$, K.M. Ramey, and F. Heidet ${ }^{1}$ \\ ${ }^{1}$ Argonne National Laboratory \\ 9700 South Cass Ave, Lemont IL 60439
}

The submitted manuscript has been created by UChicago Argonne, LLC, Operator of Argonne National Laboratory ("Argonne"). Argonne, a U.S. Department of Energy Office of Science laboratory, is operated under Contract No. DE-AC02-06CH11357. The U.S. Government retains for itself, and others acting on its behalf, a paid-up nonexclusive, irrevocable worldwide license in said article to reproduce, prepare derivative works, distribute copies to the public, and perform publicly and display publicly, by or on behalf of the Government. The Department of Energy will provide public access to these results of federally sponsored research in accordance with the DOE Public Access Plan. http://energy.gov/downloads/doe-public-access-plan 


\title{
IMPACT OF NUCLEAR DATA LIBRARIES ON PREDICTED FAST REACTOR PERFORMANCE
}

\author{
A.G. Nelson ${ }^{1}$, K.M. Ramey ${ }^{2}$, and F. Heidet ${ }^{1}$ \\ ${ }^{1}$ Argonne National Laboratory \\ 9700 South Cass Ave, Lemont, IL 60439 \\ ${ }^{2}$ Georgia Institute of Technology \\ 770 State St. NW, Atlanta, GA 30313 \\ agnelson@anl.gov,kmramey@gatech.edu, fheidet@anl.gov
}

\begin{abstract}
The nuclear data evaluation process inherently yields a nuclear data set designed to produce accurate results for the neutron energy spectra corresponding to a specific benchmark suite of experiments. When studying reactors with spectral conditions outside of, or not well represented by, the experimental database used to evaluate the nuclear data, care should be given to the relevance of the nuclear data used. In such cases, larger biases or uncertainties may be present than in a reactor with well-represented spectra. The motivation of this work is to understand the magnitude of differences between recent nuclear data libraries to provide estimates for expected variability in criticality and power distribution results for sodiumcooled, steel-reflected, metal-fueled fast reactor designs.

This work was specifically performed by creating a 3D OpenMC model of a sodium-cooled, steel-reflected, metal-fueled fast reactor similar to the FASTER design but without a thermal test region. This OpenMC model was used to compare the differences in eigenvalues, reactivity coefficients, and the spatial and energetic effects on flux and power distributions between the ENDF/B-VII.0, ENDF/B-VII.1, ENDF/B-VIII.0, JEFF-3.2, and JEFF-3.3 nuclear data libraries. These investigations have revealed that reactivity differences between the above libraries can vary by nearly $900 \mathrm{pcm}$ and the fine-group fluxes can vary by up to $18 \%$ in individual groups. Results also show a strong variation in the flux and power distributions near the fuel/reflector interface due to the high variability in the ${ }^{56} \mathrm{Fe}$ cross sections in the libraries examined. This indicates that core design efforts of a sodium-cooled, steel-reflected, metalfueled reactor will require the application of relatively large nuclear data uncertainties and/or the development of a representative benchmark-quality experiment.
\end{abstract}

KEYWORDS: ENDF, JEFF, Fast Reactor, FASTER, Nuclear Data

\section{INTRODUCTION}

Having a reliable nuclear data set is imperative for obtaining accurate results from models and simulations of any nuclear system. Inaccurate data due to limited testing or energy resolution can lead to large uncertainties and potential biases. This is especially relevant today as the next generation of reactor technologies can operate with materials and neutron energy spectra different from the majority of current reactors. The nuclear data evaluation process takes experimental results for a variety of neutron energy spectra to produce a data set that is accurate for systems with parameters similar to those of the suite of 
critical experiments used to develop the data set. There is no guarantee that data for systems with different material compositions and neutron spectra that deviate from the experimental conditions considered will provide reliable results. Further, one would expect data sets to have larger uncertainties for these systems than those matching the experiments, as well as be susceptible to biases from potentially inaccurate interpolations and extrapolations.

The focus of this work is to compare nuclear data libraries in order to identify and quantify the sources of inconsistencies across these libraries specifically for fast-spectrum, sodium-cooled, steel-reflected, metalfueled reactor designs. This can support the design and deployment of similar reactors, for example the Versatile Test Reactor (VTR) [1]. This work was performed to provide perspective on the degree of variability associated with the data validation process for the reactor type of interest. Such perspective is useful to inform design reactivity uncertainties until more rigorous analyses are complete.

The nuclear data libraries considered in this work are: ENDF/B-VII.0, ENDF/B-VII.1, ENDF/B-VIII.0, JEFF-3.2, and JEFF-3.3 [2-6]. Isotopes of interest include ${ }^{52} \mathrm{Cr},{ }^{56} \mathrm{Fe},{ }^{238} \mathrm{U}$, and others. Data libraries are compared using results from a modified version of the FASTER test reactor design [7]. All simulations used the 3D Monte Carlo code OpenMC [8] version 0.10.0.

\section{BACKGROUND}

Accurate isotopic interaction data is necessary to achieve high confidence in modeling with reduced reliance on specific experiments representing the specific configuration of interest. No matter the robustness of the methods used- geometric resolution of features, order of discretization for deterministic solvers, or number of particles used in Monte Carlo - if the underlying nuclear data has large uncertainties and biases, the results will also suffer the same limitations. Nuclear data evaluations are often inferred from measured results of controlled benchmark experiments. While these systems have well-characterized compositions and observable quantities (neutron, gamma, and fission product yields, etc.), it is often difficult to attribute individual reactions to individual isotopes. This creates a correlation within the system whereby some reactions may be overestimated while others are underestimated, but produces the correct integral effect for the composition and spectrum of the experiment.

One of the consequences of this process is that data is most reliable for modeling systems and conditions similar to those used in the validation experiments. Any error contributions from individual isotope reactions are correlated and can effectively cancel due to the compensation of these errors [9]. If specific materials, isotopes, or the flux spectra change from the validation experiments, then the magnitude of these errors can change. Therefore, the applicability of nuclear data should be verified, especially when moving away from the conditions of the reference experiments [10]. This is the case for many proposed advanced reactor concepts currently being pursued around the world, including sodium fast reactor (SFR) designs. Studies have shown that uncertainty in existing data could results in eigenvalue discrepancies on the order of thousands of pcm [11]. To address this, data consortia have identified isotopes and reaction channels in most need of improvement [12], and some of these have already been updated in recent nuclear data library releases [13]. This work investigates the magnitude of this effect to support design activities for the VTR.

\section{METHODOLOGY}

The model used to conduct various cross section sensitivity studies is based on the FASTER test reactor design [7] with some significant modifications. FASTER is a sodium-cooled fast spectrum test reactor which is able to provide both fast and thermal neutron flux to test locations in the core. The focus of this paper is on fast-spectrum systems, so the thermal test section of the FASTER design was replaced. Figure 1 provides the resulting radial core layout. Each of the eight radial active core assembly types are homogenized. Axial reflecting regions are present approximately $130 \mathrm{~cm}$ both above and below the $80 \mathrm{~cm}$ 
tall core. All components of the core are assumed to be at $294 \mathrm{~K}$ as Doppler effects are beyond the scope of this work. While not realistic from a reactor design perspective, this temperature also corresponds to a reference temperature in all considered nuclear data libraries; therefore, no temperature-dependent data interpolation was necessary.

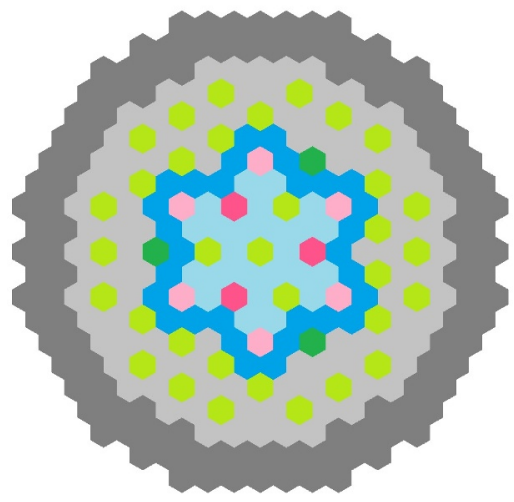

\author{
Number of Assemblies \\ Inner Core (24) \\ Outer Core (30) \\ Primary Control (6) \\ Secondary Control (3) \\ Reflector (99) \\ Shield (114) \\ Fast Test Location (34) \\ Fast Closed Loop (3) \\ Total Assemblies (313)
}

Figure 1. FASTER-like SFR Core Layout.

The metallic fuel used in the modified FASTER design is a U-19w\%Pu- $6 \mathrm{w} \% \mathrm{Zr}$ alloy. The uranium is of natural enrichment, and because the fuel is $75 \mathrm{w} \%$ uranium, ${ }^{238} \mathrm{U}$ makes up a majority of the fuel. ${ }^{239} \mathrm{Pu}$ is the primary fissile isotope; the plutonium reactor-grade, which differs from the weapons-grade proposed in the actual FASTER design. There are three reasons for the change: firstly, to offset the reactivity gained from moving from a mixed-spectrum to fast system; secondly, to better capture mid-cycle behaviors with higher contributions from isotopes of plutonium other than ${ }^{239} \mathrm{Pu}$; and thirdly, to better agree with previous material analyses of metallic U-Pu-Zr fuel [14]. The fuel cladding and other structural components are composed of HT-9 stainless steel [15]. This results in $\sim 22$ vol. \% of the core being HT-9; the two primary isotopes of interest are ${ }^{56} \mathrm{Fe}(91.8 \mathrm{a} \%$ natural abundance of iron $)$ and ${ }^{52} \mathrm{Cr}(83.8 \mathrm{a} \%$ natural abundance of chromium). The coolant ( $\sim 28 \mathrm{vol} . \%$ of the core) of the system is sodium, which only has one naturally occurring isotope of ${ }^{23} \mathrm{Na}$. In review, the most prevalent isotopes in the core are ${ }^{23} \mathrm{Na},{ }^{52} \mathrm{Cr},{ }^{56} \mathrm{Fe},{ }^{238} \mathrm{U}$, and ${ }^{239} \mathrm{Pu}$. As will be shown in Sections 4.2 and 4.3.3, these isotopes are also responsible for a majority of the observed differences between the nuclear data libraries.

\title{
4. RESULTS
}

The following results come from making comparisons between nuclear data libraries either as a whole or by substituting individual isotopes from the library of interest into some other reference library. Initially, whole data libraries are changed to gain perspective on the magnitude of differences one can expect. Substitution of individual isotope data from one library to a reference library is then done to identify isotopes driving differences between data libraries and quantify their impact. The spatial dependence of any observed differences are investigated to gain an understanding on the nuclear data impacts to the predicted power distribution and irradiation test conditions.

\subsection{Whole Nuclear Data Libraries Differences}

The core layout shown in Figure 1, with all control assemblies fully withdrawn, was simulated in OpenMC with the five considered nuclear data libraries of ENDF/B-VII.0, ENDF/B-VII.1, ENDF/B-VIII-0, JEFF3.2, and JEFF-3.3. Eigenvalue differences between the simulations are provided in Table I. The largest observed reactivity difference is between ENDF/B-VII.1 and JEFF-3.3 of $\sim 900$ pcm. Group-wise flux differences over the fueled core region between the reference ENDF/B-VII.1 library and the other four 
libraries for all isotopes present in the model are shown in Figure 2. Most of the sharp differences occur near strong resonances for various isotopes present in the core. It should be noted that larger group-wise flux differences are observed when moving away from the active core region such as in the radial reflector region.

Table I. Eigenvalue differences: Comparison of nuclear data libraries.

\begin{tabular}{|c|c|c|}
\hline Library & keff & $\boldsymbol{\Delta} \boldsymbol{\rho}[\mathbf{p c m}]$ \\
\hline ENDF/B-VII.1 & $1.05234 \pm 0.00001$ & 0 (reference) \\
\hline ENDF/B-VII.0 & $1.05600 \pm 0.00001$ & $348 \pm 2$ \\
\hline ENDF/B-VIII.0 & $1.05381 \pm 0.00001$ & $140 \pm 2$ \\
\hline JEFF-3.2 & $1.05916 \pm 0.00001$ & $648 \pm 2$ \\
\hline JEFF-3.3 & $1.06190 \pm 0.00001$ & $908 \pm 2$ \\
\hline
\end{tabular}

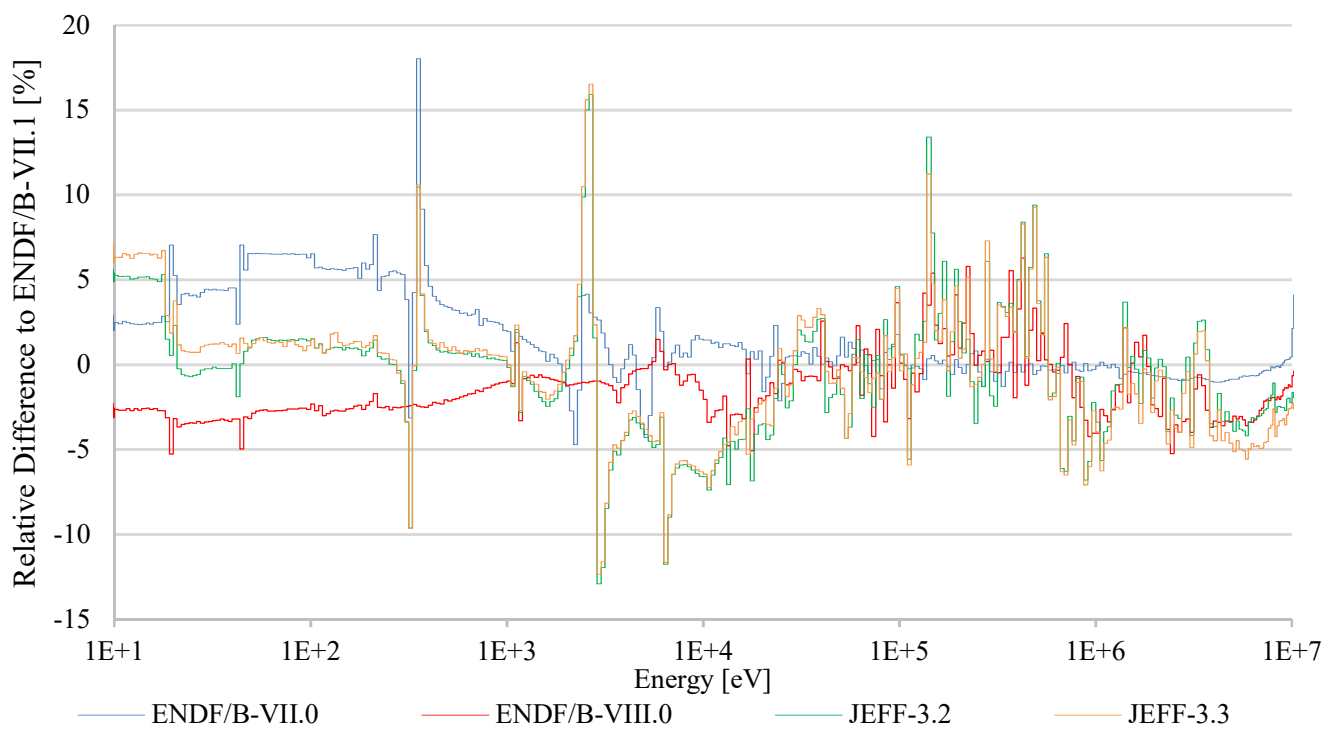

Figure 2. Relative Group-Wise Flux Differences over Active Core Region.

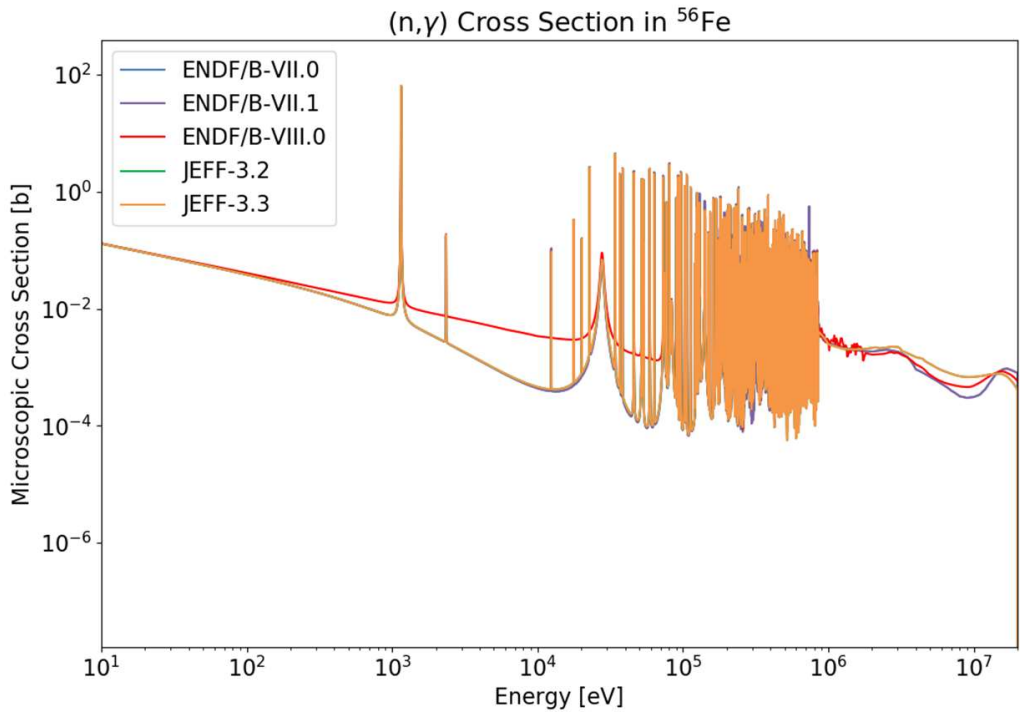

Figure 3. (n, $\gamma)$ Capture Cross Section of ${ }^{56} \mathrm{Fe}$ for Considered Nuclear Data Libraries. 


\subsection{Substitution of Individual Isotopes}

Isotopes of an element can have compensating errors whereby individual isotope listings may have significant differences from reality but are balanced by complimentary differences in the other isotopes. For this reason, isotopic substitution between nuclear data libraries cannot guarantee that observed differences are purely attributable to the isotope of interest since the other isotopes of the element will still have their own errors which are now uncompensated when individually substituted. Despite these limitations, isotopic substitutions can be useful for qualitatively identifying discrepant isotopes and elements in a system. Large differences would reflect that either the whole element has issues or that the isotopic splits within the element are biased despite being consistent when used together.

ENDF/B-VII.1 was used as the reference library for conducting isotopic substitutions. Each substitution is performed by substituting the data for the specified isotope from the specified nuclear data library in place of the corresponding isotopic data from ENDF/B-VII.1. Reactivity differences from conducting an isotopic substitution for meaningful isotopes in the simplified FASTER model are provided in Table II. Each entry of Table II has a statistical uncertainty of about $5 \mathrm{pcm}$. Notable differences include: ${ }^{52} \mathrm{Cr}$ of ENDF/B-VII.0 with $\sim 250 \mathrm{pcm}$ difference, ${ }^{56} \mathrm{Fe}$ of ENDF/B-VIII.0 with $\sim 370 \mathrm{pcm}$ difference, and ${ }^{238} \mathrm{U}$ of JEFF-3.2 with $\sim 450 \mathrm{pcm}$ difference. The difference in reactivity when using ENDF-B/VIII.0's evaluation of ${ }^{56} \mathrm{Fe}$ is readily apparent when viewing the capture cross sections of this isotope across the libraries of interest in Figure 3. Many of the substitutions produced small or statistically insignificant reactivity deviations. The list of isotopes with reactivity differences below $20 \mathrm{pcm}$ that are not shown in Table II includes: ${ }^{10} \mathrm{~B},{ }^{11} \mathrm{~B},{ }^{12} \mathrm{C},{ }^{13} \mathrm{C}$, ${ }^{50} \mathrm{Cr},{ }^{54} \mathrm{Cr},{ }^{92} \mathrm{Mo},{ }^{94} \mathrm{Mo},{ }^{95} \mathrm{Mo},{ }^{96} \mathrm{Mo},{ }^{97} \mathrm{Mo},{ }^{98} \mathrm{Mo},{ }^{100} \mathrm{Mo},{ }^{60} \mathrm{Ni},{ }^{61} \mathrm{Ni},{ }^{62} \mathrm{Ni},{ }^{64} \mathrm{Ni},{ }^{31} \mathrm{P},{ }^{242} \mathrm{Pu},{ }^{32} \mathrm{~S},{ }^{33} \mathrm{~S},{ }^{34} \mathrm{~S},{ }^{36} \mathrm{~S}$, ${ }^{28} \mathrm{Si},{ }^{29} \mathrm{Si},{ }^{30} \mathrm{Si},{ }^{50} \mathrm{~V},{ }^{180} \mathrm{~W},{ }^{182} \mathrm{~W},{ }^{183} \mathrm{~W},{ }^{184} \mathrm{~W},{ }^{186} \mathrm{~W},{ }^{91} \mathrm{Zr},{ }^{94} \mathrm{Zr}$, and ${ }^{96} \mathrm{Zr}$.

Table II. Isotopic Substitutions: Reactivity differences [pcm] from reference ENDF/B-VII.1 result.

\begin{tabular}{|c|c|c|c|c|}
\hline Isotope & ENDF/B-VII.0 & ENDF/B-VIII.0 & JEFF-3.2 & JEFF-3.3 \\
\hline${ }^{23} \mathrm{Na}$ & 5 & 25 & 64 & 55 \\
\hline${ }^{90} \mathrm{Zr}$ & 18 & 1 & 115 & 34 \\
\hline${ }^{92} \mathrm{Zr}$ & 10 & 11 & 36 & 29 \\
\hline${ }^{235} \mathrm{U}$ & 6 & -2 & 0 & -24 \\
\hline${ }^{23} \mathrm{U}$ & 3 & 181 & -16 & 445 \\
\hline${ }^{239} \mathrm{Pu}$ & 8 & 60 & -50 & -177 \\
\hline${ }^{240} \mathrm{Pu}$ & 68 & 141 & 28 & 28 \\
\hline${ }^{241} \mathrm{Pu}$ & 8 & 1 & 41 & 22 \\
\hline${ }^{51} \mathrm{~V}$ & 12 & 3 & 13 & 25 \\
\hline${ }^{52} \mathrm{Cr}$ & 248 & 2 & 20 & 50 \\
\hline${ }^{53} \mathrm{Cr}$ & -32 & -4 & 14 & 10 \\
\hline${ }^{55} \mathrm{Mn}$ & 44 & -4 & 1 & -2 \\
\hline${ }^{54} \mathrm{Fe}$ & -1 & 86 & 49 & 46 \\
\hline${ }^{56} \mathrm{Fe}$ & -2 & -372 & 165 & 172 \\
\hline${ }^{57} \mathrm{Fe}$ & 2 & 13 & 137 & 141 \\
\hline${ }^{58} \mathrm{Fe}$ & 2 & 10 & 26 & 30 \\
\hline${ }^{58} \mathrm{Ni}$ & 170 & -6 & -8 & -12 \\
\hline
\end{tabular}

\subsection{Geometric Effects}

Spatially-dependent behaviors are relevant in a nuclear data library comparison because they can inform the impact of material differences and spectral changes within a model. This section addresses some of the geometric effects observed from switching libraries. While this work focused predominantly on radial 
behaviors, the axial effects would behave similarly to the radial ones since comparable compositions surround the fueled core region in every direction (mostly structural stainless steel and liquid sodium coolant). While not discussed in its own section, all five data libraries show good agreement on the control assembly differential reactivity worth with the maximum observed difference in rod worth of $\sim 1.3 \%$ occurring between JEFF-3.2 and JEFF-3.3. This indicates that the choice of library does not significantly alter the total neutron absorption of the primary absorber within the $\mathrm{B}_{4} \mathrm{C}$ control rod, ${ }^{10} \mathrm{~B}$ due to a similarity for all sets of the energy spectra impingent on the control rods, the control rod moderation, and the control rod absorption cross sections.

\subsubsection{Radial flux differences}

One of the most applicable parameters affected by switching nuclear data libraries is the change to the neutron flux as this affects many downstream design parameters. To this end, Figure 4 shows the relative difference of the one-group flux for each of the other four libraries of interest compared to ENDF/B-VII.1. The statistical uncertainty is $\sim 0.03 \%$ in the middle of the core and increases up to $\sim 0.5 \%$ at the periphery of the shield.

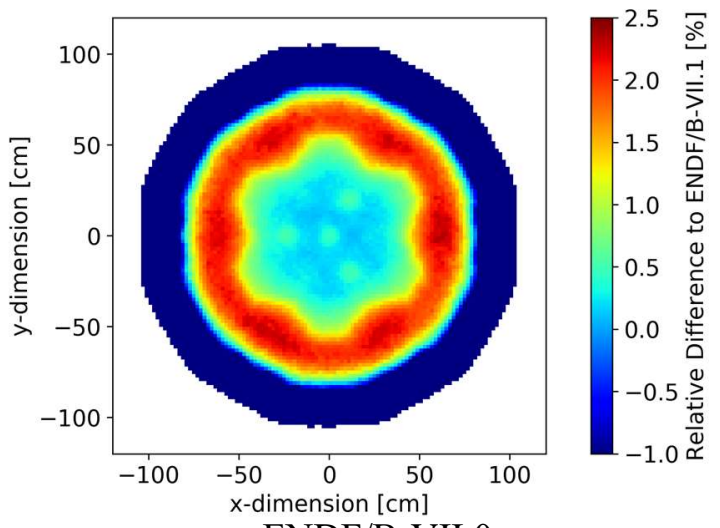

ENDF/B-VII.0

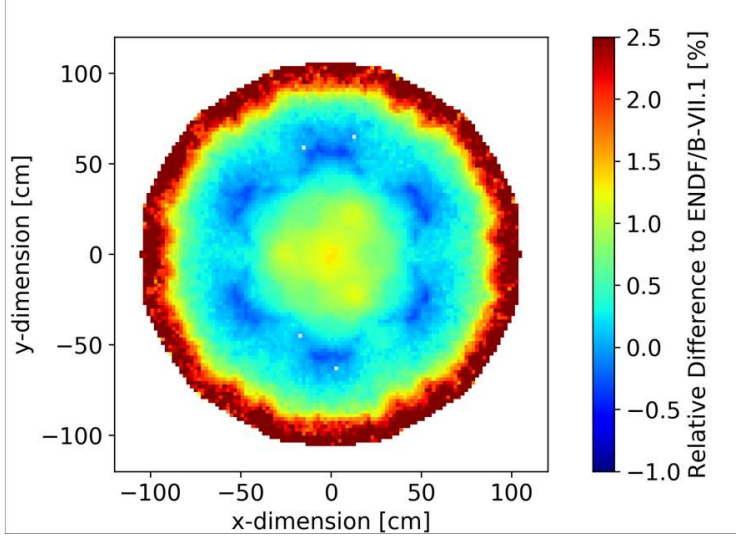

JEFF-3.2
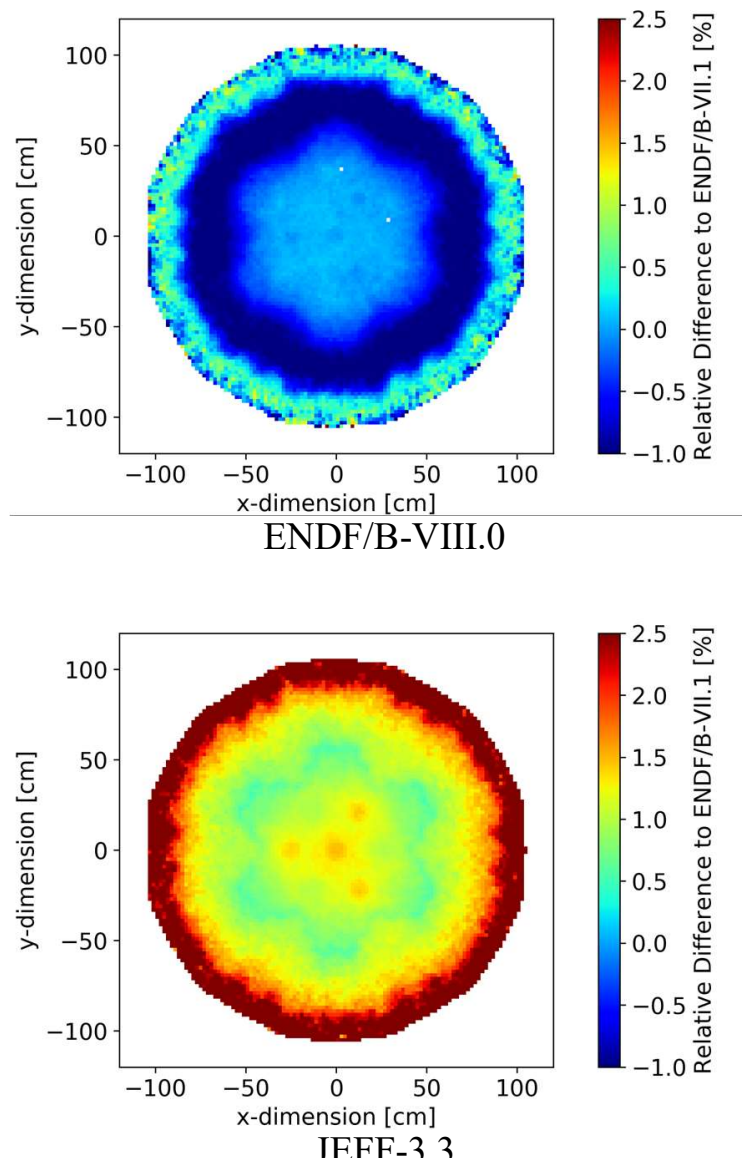

JEFF-3.3

Figure 4. One-group Flux Relative Differences from Reference ENDF/B-VII.1 Results.

Generally, the largest discrepancies of Figure 4 are near the reflector itself, the fuel-reflector interfaces, and the shielded region. The central fueled region has the most consistent performance in each of the libraries with the largest difference occurring between ENDF/B-VIII.0 and JEFF-3.3 of $\sim 2 \%$. The reflector region 
between the inner fueled and outer shield regions has a peak difference between ENDF/B-VII.0 and ENDF/B-VIII.0 of $\sim 3.5 \%$. The overall largest observed differences occur at the periphery of the model in the shield region. The maximum differences of both ENDF/B-VII.0 and JEFF-3.3 exceed the scale of the plots which were chosen to emphasize trends in all four comparisons. ENDF/B-VII.0 differs by as much as $-7 \%$ in the shield and JEFF-3.3 differs by just over 3\% from ENDF/B-VII.1 in the shield. If considered with respect to each other, ENDF/B-VII.0 and JEFF-3.3 diverge on the one-group flux by more than $10 \%$ in certain locations of the shield. This discrepancy was not investigated further as this work is more concerned with in-core behavior; however, these differences may be important for shielding or ex-core detector-related analyses.

\subsubsection{Radial fission rate differences}

Another important parameter is the power distribution, a parameter that is key to evaluating the thermal performance of a reactor. To identify the impacts of nuclear data libraries on the power distributions, OpenMC was used to generate the fission rate distributions. The resultant differences in fission rates between the libraries of interest are provided in Figure 5. The statistical uncertainty is $\sim 0.04 \%$ near the center of the fueled region and increases up to $\sim 0.1 \%$ at the edge of the peripheral fuel assemblies.

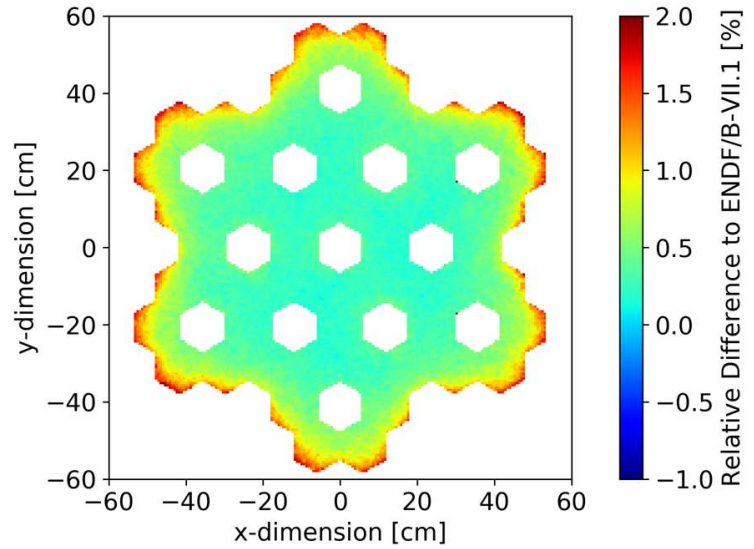

ENDF/B-VII.0

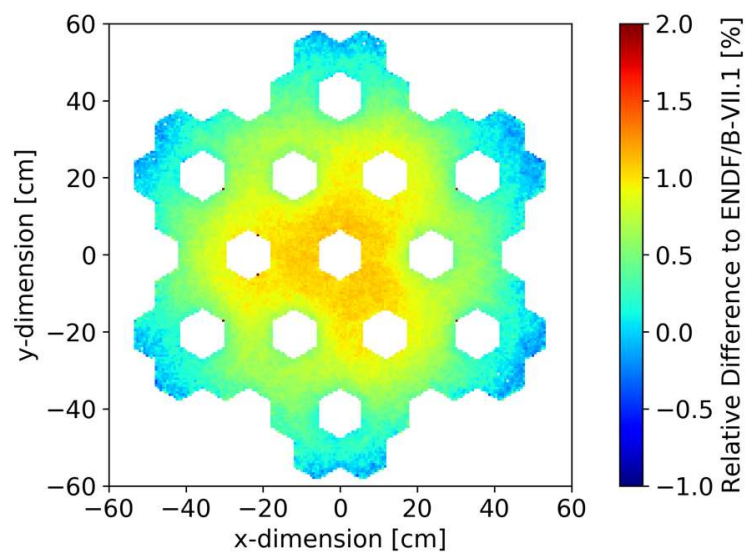

JEFF-3.2

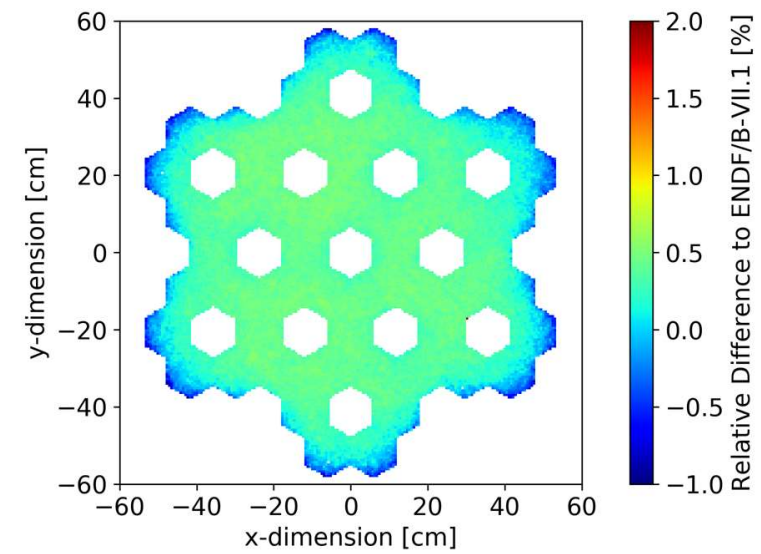

ENDF/B-VIII.0

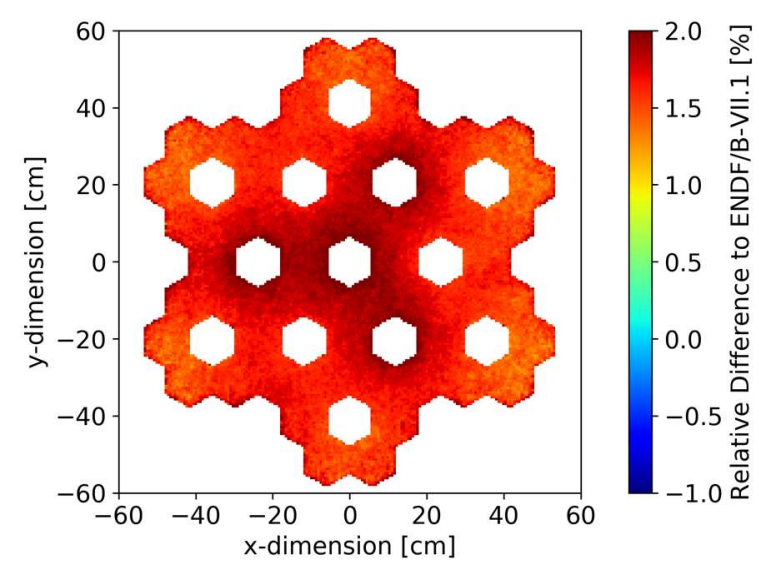

JEFF-3.3

Figure 5. Fission Rate Relative Differences from Reference ENDF/B-VII.1 Results. 
While all libraries show a slight increase in fission rate compared to ENDF/B-VII.1, the largest difference is seen in JEFF-3.3. This is expected since the JEFF-3.3 library has largest eigenvalue for this case, as shown in Table I. Another observation is that the fission rate differences decrease in the peripheral fuel assemblies for ENDF/B-VIII.0, JEFF-3.2, and JEFF.3.3. The exception to this is ENDF/B-VII.0, which sees an increase in the fission rate differences along the periphery.

\subsubsection{Radial one-group cross section differences}

To further evaluate the sources of the reactivity, flux, and power differences, this subsection discusses the changes to the macroscopic cross sections in the fuel, reflector, and shield due to performing the isotopic substitutions introduced earlier. Four macroscopic cross sections (scattering, absorption, (n, $\gamma$ ) capture, and fission) are considered in three material regions (fuel, reflector, and shield). Of all the $\sim 50$ isotopes used in the model, the ones differing by more than $0.25 \%$ from the reference ENDF/B-VII.1 value for a macroscopic cross section are presented in Table III. Statistical uncertainties for cross section differences are less than $0.01 \%$ for each entry.

Table III. Cross section impact due to isotopic substitution: Relative percent difference in scattering, absorption, $(\mathrm{n}, \gamma)$ capture, and fission macroscopic cross section from isotopic substitutions in the reference ENDF/B-VII.1 library. Note: differences greater than $0.25 \%$ are bold.

\begin{tabular}{|c|c|c|c|c|c|c|c|c|c|c|}
\hline & \multicolumn{4}{|c|}{ Fuel } & \multicolumn{3}{|c|}{ Reflector } & \multicolumn{3}{|c|}{ Shield } \\
\hline & $\Delta \Sigma_{\mathrm{s}}$ & $\Delta \Sigma_{\mathrm{a}}$ & $\Delta \Sigma_{\gamma}$ & $\Delta \Sigma_{\mathrm{f}}$ & $\Delta \Sigma_{\mathrm{s}}$ & $\Delta \Sigma_{\mathrm{a}}$ & $\Delta \Sigma_{\gamma}$ & $\Delta \Sigma_{\mathrm{s}}$ & $\Delta \Sigma_{\mathrm{a}}$ & $\Delta \Sigma_{\gamma}$ \\
\hline \multicolumn{11}{|l|}{ ENDF/B-VII.0 } \\
\hline${ }^{52} \mathrm{Cr}$ & 0.52 & 0.31 & 0.63 & 0.07 & 1.94 & 1.92 & 1.93 & 0.34 & 1.16 & 1.59 \\
\hline${ }^{53} \mathrm{Cr}$ & -0.03 & -0.03 & -0.08 & 0.00 & 0.19 & 0.38 & 0.39 & 0.01 & -0.28 & 0.35 \\
\hline${ }^{55} \mathrm{Mn}$ & 0.00 & -0.03 & -0.07 & 0.01 & 0.12 & -0.58 & -0.58 & -0.02 & -0.16 & -0.67 \\
\hline${ }^{58} \mathrm{Ni}$ & -0.16 & -0.29 & -0.96 & 0.14 & -0.17 & -0.26 & -0.26 & -0.01 & -0.16 & -0.01 \\
\hline${ }^{240} \mathrm{Pu}$ & -0.04 & -0.06 & -0.27 & 0.11 & -0.03 & -0.06 & -0.06 & 0.00 & -0.01 & -0.02 \\
\hline \multicolumn{11}{|l|}{ ENDF/B-VIII.0 } \\
\hline${ }^{10} \mathrm{~B}$ & 0.00 & 0.01 & 0.01 & 0.00 & 0.00 & 0.00 & 0.00 & -0.02 & 0.03 & -0.66 \\
\hline${ }^{54} \mathrm{Fe}$ & 0.04 & -0.01 & 0.03 & -0.04 & 0.06 & 0.04 & 0.05 & 0.06 & 0.36 & 0.74 \\
\hline${ }^{56} \mathrm{Fe}$ & -0.02 & -0.20 & 0.00 & -0.34 & -0.79 & 2.85 & 2.91 & -0.03 & -0.76 & 8.34 \\
\hline${ }^{57} \mathrm{Fe}$ & 0.00 & 0.06 & 0.14 & 0.00 & -0.11 & 0.56 & 0.56 & 0.14 & 1.14 & 1.15 \\
\hline${ }^{238} \mathrm{U}$ & -0.16 & -0.29 & -0.95 & 0.15 & -0.15 & -0.26 & -0.26 & -0.01 & -0.15 & -0.11 \\
\hline \multicolumn{11}{|l|}{ JEFF-3.2 } \\
\hline${ }^{23} \mathrm{Na}$ & -0.22 & -0.15 & -0.13 & -0.16 & -0.02 & 0.03 & 0.03 & 0.06 & 0.41 & 0.62 \\
\hline${ }^{56} \mathrm{Fe}$ & -0.01 & -0.38 & -0.89 & 0.01 & -0.21 & -1.94 & -1.95 & -0.29 & -1.57 & -2.12 \\
\hline${ }^{57} \mathrm{Fe}$ & 0.23 & 0.19 & 0.39 & 0.04 & 0.60 & 0.99 & 0.99 & 0.35 & 1.97 & 0.59 \\
\hline${ }^{90} \mathrm{Zr}$ & 1.00 & 0.13 & 0.32 & -0.01 & 0.10 & 0.20 & 0.20 & 0.00 & 0.05 & 0.12 \\
\hline${ }^{186} \mathrm{~W}$ & 0.00 & 0.01 & 0.01 & 0.02 & 0.09 & -0.18 & -0.18 & 0.00 & 0.04 & -0.30 \\
\hline${ }^{238} \mathrm{U}$ & -0.08 & $\begin{array}{l}-0.59 \\
\end{array}$ & -1.15 & -0.16 & -0.12 & -0.26 & -0.25 & 0.02 & -0.03 & -0.10 \\
\hline${ }^{239} \mathrm{Pu}$ & 0.06 & 0.11 & 0.82 & -0.44 & -0.09 & -0.14 & -0.14 & -0.02 & -0.10 & -0.08 \\
\hline${ }^{240} \mathrm{Pu}$ & -0.06 & 0.45 & 0.42 & 0.47 & -0.03 & -0.04 & -0.04 & -0.01 & -0.05 & 0.00 \\
\hline \multicolumn{11}{|l|}{ JEFF-3.3 } \\
\hline${ }^{23} \mathrm{Na}$ & -0.22 & -0.15 & -0.14 & -0.16 & -0.01 & 0.03 & 0.03 & 0.06 & 0.39 & 0.61 \\
\hline${ }^{51} \mathrm{~V}$ & 0.06 & 0.04 & 0.06 & 0.01 & 0.28 & $\mathbf{0 . 3 0}$ & 0.30 & 0.04 & 0.03 & -0.01 \\
\hline${ }^{56} \mathrm{Fe}$ & -0.01 & $\begin{array}{l}-0.38 \\
\end{array}$ & $\begin{array}{l}-0.88 \\
\end{array}$ & 0.00 & -0.20 & -1.94 & -1.95 & -0.29 & -1.57 & -2.14 \\
\hline${ }^{57} \mathrm{Fe}$ & 0.23 & 0.19 & 0.40 & 0.04 & 0.60 & 1.00 & 1.01 & 0.35 & 1.96 & 0.72 \\
\hline${ }^{238} \mathrm{U}$ & 0.08 & -1.23 & -2.42 & -0.36 & -0.01 & -0.02 & 0.00 & 0.03 & 0.04 & -0.01 \\
\hline${ }^{239} \mathrm{Pu}$ & -0.15 & -0.06 & -0.68 & 0.41 & -0.08 & -0.14 & -0.13 & 0.00 & -0.05 & -0.04 \\
\hline${ }^{240} \mathrm{Pu}$ & -0.06 & 0.45 & 0.42 & 0.47 & -0.02 & -0.04 & -0.04 & 0.00 & -0.02 & 0.04 \\
\hline
\end{tabular}


Chromium is the main driver between ENDF/B-VII.0 and ENDF/B-VII.1 differences with ${ }^{52} \mathrm{Cr}$ being the dominant contributor. Iron is the source of a majority of the difference between ENDF/B-VII.1 and ENDF/B-VIII.0, as expected given the capture cross section differences shown in Figure 3. While $\sim 92 \%$ of iron is ${ }^{56} \mathrm{Fe}$, some of the other isotopes are also significant. This is likely due to compensating effects between the isotopes of iron. Both JEFF libraries also have large differences with ENDF/B-VII.1 due to iron. JEFF-3.3 specifically exhibits large differences with ${ }^{238} \mathrm{U}$ data, particularly with the $(\mathrm{n}, \gamma)$ capture cross section at $\sim 2.4 \%$. A concern of this is that it will affect predicted plutonium production and hence impact performance predictions with depletion as well as fuel cycle analyses for this type of reactor.

The cross section differences of Table III are able to explain a majority of the reactivity differences observed in Table II. In Table II, ENDF/B-VII.0 ${ }^{52} \mathrm{Cr}$ contributed a significant eigenvalue bias; Table III shows that the largest differences in cross sections due to this isotope occur in the reflector. ENDF/B-VIII.0, JEFF3.2, and JEFF-3.3 all have a similar explanation for their differences due to iron. Each library has the smallest difference inside the fueled region of the core with larger differences in both the reflector and shield regions. This is especially evident in the case of ENDF/B-VIII.0, which shows relatively good agreement within the fuel region despite having $(n, \gamma)$ capture differences near $3 \%$ in the reflector and more than $8 \%$ differences in the shield.

\subsubsection{Radial fission parameters in simplified model}

In order to simplify the evaluation of the effects of the usage of one complete library over another, a simplified model was developed which preserves the same spectral features of the original model. The original model was simplified to a concentric cylindrical one (Figure 6) with axial reflectors (red and dark blue) similar to those of the modified FASTER model in Figure 1 and employing volumetric homogenization within the appropriate cylindrical regions. This model allows for easier partitioning of the core in the fuel region and more straightforward presentation of results.

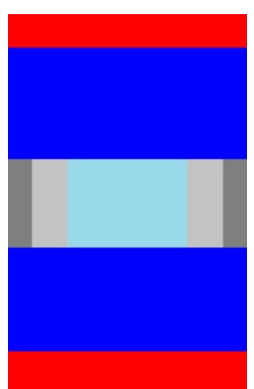

Axial View

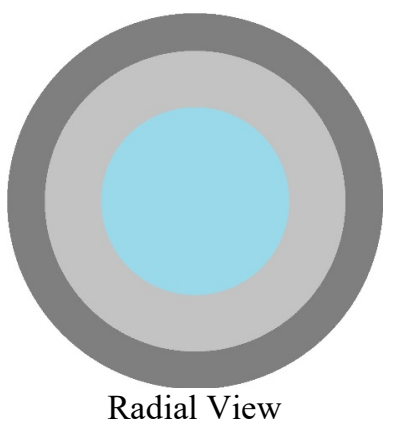

Radial View

\begin{tabular}{|c|c|}
\hline Region & Radius [cm] \\
\hline Fuel & 55 \\
\hline Reflector & 88 \\
\hline Shield & 110 \\
\hline
\end{tabular}

Dimensions

Figure 6. Geometry of Simplified Model for Radial Study.

The cylindrical model uses five equal-volume tallies in the homogenized fuel region with each of the nuclear data libraries of interest. Four neutronic parameters are considered in each tally region: fission rate, $v$-fission rate, neutron production $(v)$, and the total delayed neutron fraction $(\beta)$. Note that this study only evaluated the total delayed neutron fraction since the number of delayed neutron precursor groups varies between JEFF and ENDF datasets, making comparison of the individual precursor group values of $\beta$ meaningless. Further, the value of the delayed neutron fraction is not importance-weighted. Differences of the other libraries of interest from reference ENDF/B-VII.1 results are plotted in Figure 7.

In Figure 7, the fission rate differences agree well with those observed in Figure 4. Once again, positive differences occur for each library from ENDF/B-VII.1 with a decreasing trend in ENDF/B-VIII.0, JEFF- 
3.2, and JEFF 3.3 and an increasing trend in ENDF/B-VII.0. The largest difference is with JEFF-3.3 of nearly $2 \%$ near the center of core, but this behavior is tempered when accounting for neutron production. The $v$-fission rate of JEFF-3.3 agrees more closely with the other nuclear data libraries since it has lower neutron production. Neither $v$ nor $\beta$ exhibit a strong spatial dependence. ENDF/B-VII.0, ENDF/B-VII.1, ENDF/B-VIII.0, and JEFF-3.2 all have $v$ values within $0.3 \%$ of each other, but JEFF-3.3 has a notably lower result. There is also some difference in the delayed neutron fraction, $\beta$. All three ENDF libraries agree very well on $\beta$ (less than $0.1 \%$ difference), but the JEFF libraries both report a higher value for $\beta$ by about $4 \%$. This is an important difference for safety analyses in particular where $\beta$ plays an important role in the analysis of reactor transients.
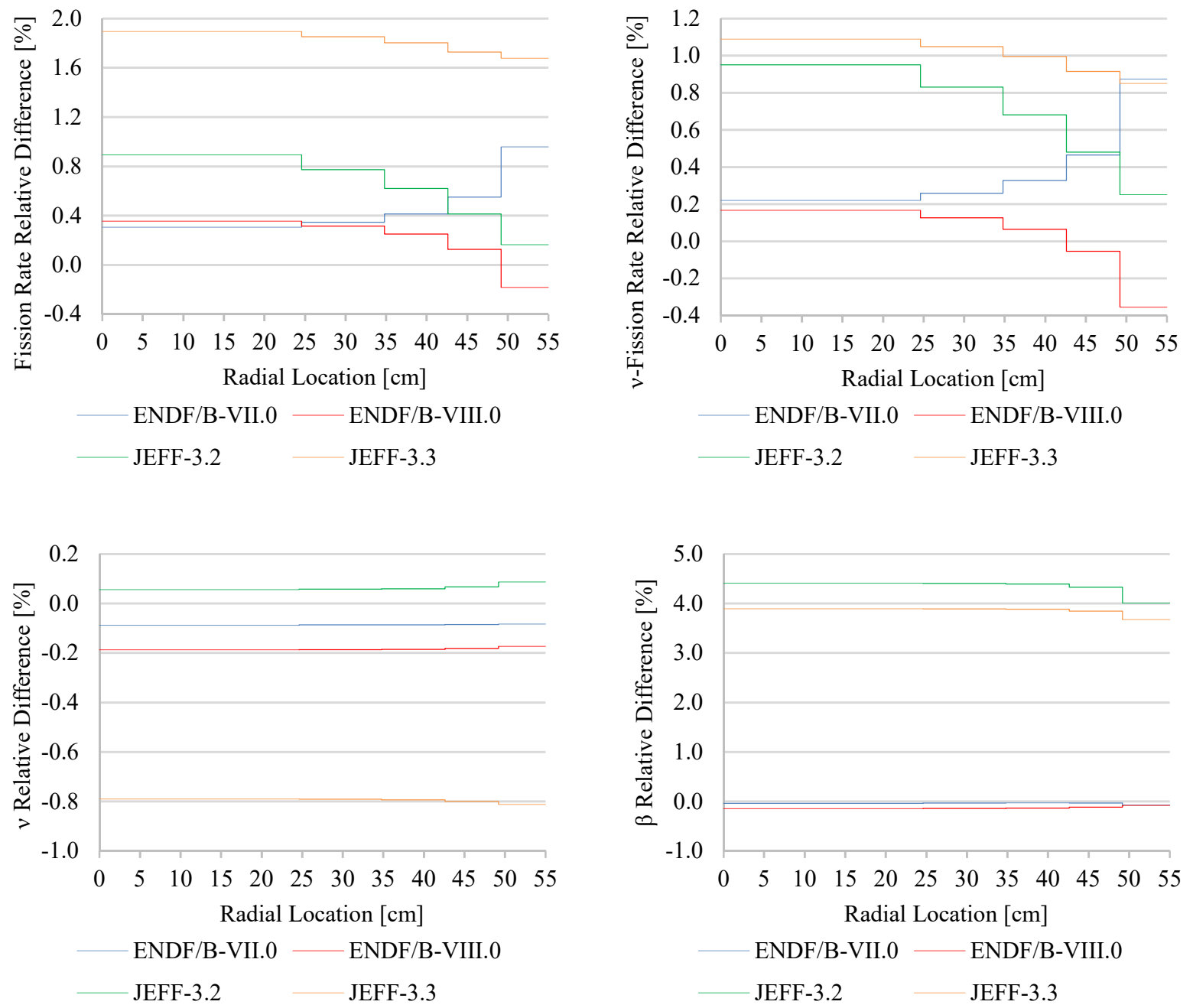

Figure 7. Radial Nuclear Data Library Differences from ENDF/B-VII.1 in One-Group Fission Rate, $v$-Fission Rate, $v$, and $\beta$ Using Simplified Cylindrical Model.

\section{CONCLUSIONS}

Reliable nuclear data is essential to accurate prediction of reactor behavior. However, applying data outside the ranges of its initial evaluation can lead to additional sources of error in the predicted performance. This is especially relevant for advanced reactor concepts which differ from conventional light water reactor 
spectra and materials. When nuclear data libraries are compiled, individual isotopes of an element have intrinsic errors from the uncertainty of attributing specific behaviors to each isotope. The integral isotopic data set for the element may match critical experimental results, but these compensating errors between the isotopes are once again only reliable for the range of neutron energy spectra and material compositions represented by the suite of integral experiments used during the evaluation process. Diverting from this reference point, errors may no longer be compensating and need to be characterized on a per-design basis.

This paper focused on nuclear data used for a sodium-cooled, steel-reflected, metal-fueled fast reactor to provide perspective on the degree of variability associated with the data validation process for such a reactor. Five nuclear data libraries were considered: ENDF/B-VII.0, ENDF/B-VII.1, ENDF/B-VIII.0, JEFF-3.2, and JEFF-3.3. First, whole libraries were used to gauge the differences between the libraries. For the case of all rods removed, the reactivity differed by up to $\sim 900 \mathrm{pcm}$. Peak multigroup differences in energy spectrum were $\sim 18 \%$ in the active core region. One-group neutron flux differed by $\sim 2 \%$ in the fuel region, $\sim 3.5 \%$ in the reflector region, and up to $10 \%$ in the shield region.

Next, the impact from individual isotopes was evaluated by substituting isotopic data from the four other libraries of interest into a reference ENDF/B-VII.1 library. The largest observed reactivity difference of $\sim 450 \mathrm{pcm}$ was between JEFF-3.2 and JEFF-3.3 for ${ }^{238} \mathrm{U}$ data. Other notable differences included chromium (mostly ${ }^{52} \mathrm{Cr}$ ) for ENDF/B-VII.0 and iron (mostly ${ }^{56} \mathrm{Fe}$ ) for all libraries. When considering one-group cross sections, these same isotopes produced the largest differences. Beyond cross sections, differences also exist in neutron production ( $\sim 0.8 \%$ between JEFF-3.2 and JEFF-3.3) and delayed neutron fraction (up to $4.5 \%$ between JEFF-3.2 and ENDF/B-VIII.0) which is significant for safety analyses.

Reactivity differences between libraries are primarily driven by the most abundant actinides ${ }^{238} \mathrm{U},{ }^{239} \mathrm{Pu}$, and ${ }^{240} \mathrm{Pu}$ ) as well as major elements in structural steel. In ENDF/B-VII.0, chromium (mostly ${ }^{52} \mathrm{Cr}$ ) is responsible for increased scattering in the reflector, returning more neutrons to the core and increasing the overall neutron economy. This is seen in Table II by the reactivity gain, in Figure 4 by the larger flux in the reflector, in Figure 5 and Figure 7 by the higher fission rate in the peripheral assemblies, and in Table III by the notable increase to the scattering cross section. Scattering differences due to iron (mostly ${ }^{56} \mathrm{Fe}$ ) have a similar effect with ENDF/B-VIII.0. In ENDF/B-VIII.0, the reactivity penalty in Table II can be justified by the large increase in $(\mathrm{n}, \gamma)$ capture in the reflector in Table III, and thus less reflector return. This behavior is not observed in the active core region which implies that the softer spectrum of the reflector has resulted in more neutrons being in the energies of the ${ }^{56} \mathrm{Fe}(\mathrm{n}, \gamma)$ cross section that have increased significantly since ENDF/B-VII.1 $[4,13]$. This is further supported by the lower flux in the reflector in Figure 4 as well as the depressed peripheral fission rate in Figure 5 despite generally flat behavior in the rest of the core. In both JEFF libraries, the same data is used for isotopes of iron but has significant differences from the ENDF libraries. JEFF-3.3 also has large differences in its ${ }^{238} U(n, \gamma)$ capture cross section, which is a major contributor to the large reactivity difference observed between JEFF-3.3 and the other libraries considered.

\section{ACKNOWLEDGMENTS}

The submitted manuscript has been created by UChicago Argonne, LLC, Operator of Argonne National Laboratory ("Argonne"). Argonne National Laboratory's work was supported by the U.S. Department of Energy, Office of Nuclear Energy under contract DE-AC02-06CH11357. The work reported in this summary is the results of R\&D studies supporting a VTR concept, cost, and schedule estimate for DOE$\mathrm{NE}$ to make a decision on procurement in the future. As such, it is pre-decisional.

\section{REFERENCES}

1. K. Pasamehmetoglu, "Versatile Test Reactor Overview," Proceedings of Advanced Reactors Summit VI, San Diego, California, January 29-31, 2019 (2019). 
2. M.B. Chadwick, et al., "ENDF/B-VII.0: Next Generation Evaluated Nuclear Data Library for Nuclear Science and Technology," Nuclear Data Sheets 107, pp. 2931-3060 (2006).

3. M.B. Chadwick, et al., "ENDF/B-VII.1: Nuclear Data for Science and Technology: Cross Sections, Covariances, Fission Product Yields and Decay Data," Nuclear Data Sheets 112, pp. 2887-2996 (2011).

4. D.A. Brown, et al., "ENDF/B-VIII.0: The $8^{\text {th }}$ Major Release of the Nuclear Reaction Data Library with CIELO-project Cross Sections, New Standards and Thermal Scattering Data," Nuclear Data Sheets 148, pp. 1-142 (2018).

5. The JEFF team. JEFF-3.2 evaluated data library. Available from: http://www.oecdnea.org/dbforms/data/eva/evatapes/jeff_32/ (2014).

6. The JEFF team. JEFF-3.3 evaluated data library. Available from: http://www.oecdnea.org/dbdata/jeff/jeff33/ (2017).

7. C. Grandy, H. Belch, J. Brunett, F. Heidet, R. Hill, E. Hoffman, E. Jin, W. Mohamed, A. Moisseytsev, S. Passerini, J. Sienicki, T. Summer, R. Vilim, S. Hayes, "FASTER Test Reactor Preconceptual Design Report," https://publications.anl.gov/anlpubs/2016/04/124963.pdf (2016).

8. P.K. Romano, N.E. Horelik, B.R. Herman, A.G. Nelson, B. Forget, K. Smith, "OpenMC: A State-ofthe-Art Monte Carlo Code for Research and Development," Annals of Nuclear Energy, 82 pp. 90-97 (2015).

9. B.T. Rearden, W.J. Marshall, J.B. Clarity, A.M. Holcomb, F. Bostelmann, J.M. Scaglione, "Initial Investigations of the Criticality Safety Validation Basis for HA-LEU Transportation," Transactions of the American Nuclear Society, Minneapolis, Minnesota, June 9-13, 2019120 pp. 517-520 (2019).

10. B.T. Rearden, F. Bostelmann, V. Sobes, A.M. Holcomb, "Overview of Nuclear Data Needs for Nuclear Energy Applications," Transactions of the American Nuclear Society, Minneapolis, Minnesota, June 913, 2019120 pp. 989-992 (2019).

11. N. Touran and J. Yang, "Sensitivities and Uncertainties Due to Nuclear Data in a Traveling Wave Reactor," Proceedings of PHYSOR 2016, Sun Valley, ID, May 1-5, 2016 (2016).

12. "Nuclear Data Needs and Capabilities for Applications," Lawrence Berkeley National Laboratory, May 27-29, 2015 https://arxiv.org/ftp/arxiv/papers/1511/1511.07772.pdf (2015).

13. M.B. Chadwick, et al., "CIELO Collaboration Summary Results: International Evaluations of Neutron Reactions on Uranium, Plutonium, Iron, Oxygen and Hydrogen," Nuclear Data Sheets 148, pp. 189213 (2018).

14. P. Manikandan, V.V. Trinadh, S. Beta, T.S.L. Narasimhan, K. Ananthasivan, M. Joseph, "Mass Spectrometric Studies on U-Pu-Zr Alloy," Journal of Nuclear Materials, 507 pp. 339-346 (2018).

15. R.G. Williams, C.J. Gesh, R.T. Pagh, "Compendium of Material Composition Data for Radiation Transport Modeling,” https://www.pnnl.gov/main/publications/external/technical_reports/PNNL15870.pdf (2006). 